\title{
COVID-19 and clinical mimics. Correct diagnosis is the key to appropriate therapy
}

\author{
Kamal Kant Sahu, ${ }^{1}$ Ajay Kumar Mishra, ${ }^{1}$ Kevin Martin, ${ }^{2}$ Iryana Chastain ${ }^{1}$ \\ ${ }^{1}$ Department of Medicine, Saint Vincent Hospital, Worcester, MA; ${ }^{2}$ Division of Pulmonology and Critical Care Medicine, \\ Reliant Medical Group, Worcester, MA, USA
}

\begin{abstract}
As of 29 April 2020, across the globe, there are 3,216,353 confirmed Coronavirus disease 2019 (COVID-19 disease) with 227,894 deaths. The health care infrastructure of most of the countries is overwhelmed due to the gigantic upsurge of the new cases within a short time period. Most of the beds in the regular wards and critical care units are currently occupied by either people under investigation (PUI) or COVID-19 confirmed cases. We hereby discuss the challenges faced while approaching any case of shortness of breath, or other common upper respiratory symptoms during the current COVID-19 pandemic era.
\end{abstract}

\section{Introduction}

The commonest clinical features that have been reported in patients presenting with COVID-19 infections are fever, cough, and shortness of breath $[1,2]$. However, these three clinical features continue to be the most common clinical presentation of various other diseases as well. Cough is commonly seen in patients with both upper and lower respiratory tract related illnesses.

Correspondence: Dr. Kamal Kant Sahu, Department of Medicine,

Saint Vincent Hospital, Worcester, MA 01608, USA.

E-mail: drkksahu85@gmail.com

Conflict of Interest: the Authors have no conflict of interest to declare.

Ethical statement: The article does not contain the participation of any human being and animal.

Authors' contributions: All the authors played a significant role in the paper. All authors have read the manuscript and agree to the content and data.

Key words: COVID-19; lung; pandemic; coronavirus.

Received for publication: 19 March 2020.

Accepted for publication: 4 May 2020.

${ }^{\circ}$ Copyright: the Author(s), 2020

Licensee PAGEPress, Italy

Monaldi Archives for Chest Disease 2020; 90:1327

doi: 10.4081/monaldi.2020.1327

This article is distributed under the terms of the Creative Commons Attribution Noncommercial License (by-nc 4.0) which permits any noncommercial use, distribution, and reproduction in any medium, provided the original author(s) and source are credited.
Shortness of breath is a common presentation in patients presenting with various pulmonary or cardiac pathologies. Amid this COVID-19 pandemic, the presence of any of these symptoms warrants testing for severe acute respiratory syndrome coronavirus 2 (SARS-CoV-2) given the high pretest probability and the grave consequence of not identifying the same in advance. The following are the three scenarios encountered by us recently, where patients were evaluated for their cardiopulmonary symptoms and were diagnosed with non-COVID-19 diseases.

\section{Case \#1}

A 53-year-old man presented with fever, shortness of breath, and cough of seven days duration. He was evaluated with radiological studies and cardiac markers. Nasopharyngeal swab for COVID-19 RT-PCR assay sent from emergency room (ER) came back negative. Computed tomography of the chest (CT chest) showed right mainstem bronchus well-circumscribed ovoid softtissue density measuring up to $1.5 \mathrm{~cm}$. The patient improved after symptomatic treatment and was planned for a repeat imaging and possibly bronchoscopy after six to eight weeks as an outpatient.

\section{Case \#2}

A 52-year-old woman presented with easy fatigability, fever, shortness of breath, and mild cough of four days duration. CT pulmonary embolism scan showed solitary pulmonary embolism involving the distal right lower lobe segmental artery. Nasopharyngeal swab for COVID-19 RT-PCR assay sent from ER came back negative. The patient improved after initiating anticoagulation.

\section{Case \#3}

An 84 -year-old man presented with fever, shortness of breath, altered sensorium of two days duration. Chest x-ray showed bilateral consolidation and blood culture grew streptococcus pneumonia. Nasopharyngeal swab for COVID-19 RT-PCR assay sent from ER came back negative. Diagnosis of community acquired pneumonia complicated with sepsis was made, that improved after initiating antibiotics.

In the above-mentioned case scenarios, all our patients presented with generic symptoms, and were evaluated for COVID-19 

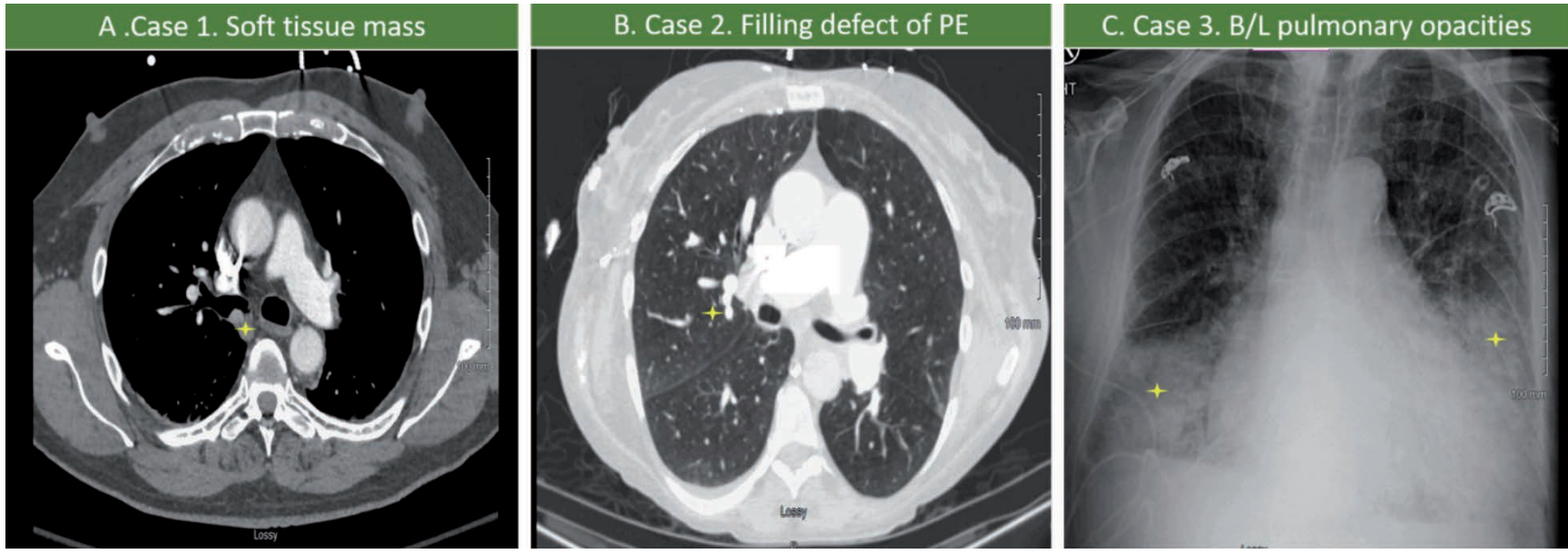

Figure 1. A) Case \#1. CT chest showed right mainstem bronchus well-circumscribed ovoid soft-tissue density measuring up to $1.5 \mathrm{~cm}$. B) Case \#2. The CT pulmonary embolism study showed solitary pulmonary embolism involving the distal right lower lobe segmental artery. C) Case \#3. Chest $\mathrm{x}$-ray showed bilateral consolidation and heterogenous opacities.

disease as well in addition to the other usual cardiopulmonary diseases. With a negative nasopharyngeal swab RT-PCR, COVID-19 was ruled out in all these patients. Patients improved after initiating respective treatment for right main bronchus lesion (Case $\# 1$ ), pulmonary embolism (Case \#2), and community acquired bacterial pneumonia (Case \#3) (Figure $1 \mathrm{~A}-\mathrm{C}$ ).

Currently, in the real world of COVID-19 pandemic era, most of the patients presenting to the emergency room or a primary care physician's office with the symptoms of fever, shortness of breath, cough are getting an initial screening of COVID-19 due to various reasons: i) presence of higher prevalence in the community, ii) international travel, iii) exposure to positive patients, iv) patients coming from rehabilitation, long term care, nursing facilities, and v) in patients with significant comorbidities including chronic obstructive pulmonary disease (COPD), congestive heart failure (CHF), coronary artery disease, chronic renal failure, chronic liver disease, and others.

The above three examples suggest that we still need to follow the dictum of "Occam's Razor" which technically means that the simplest explanation is usually the correct one in the medical practice. However, the probability of having a disease depends upon the prevalence of the particular disease in the community. With the rising number of COVID-19 cases every day, COVID-19 pneumonia is no more a "Zebra diagnosis". Also, COVID-19 pneumonia can co-exist with other common cardiopulmonary disorders. Based on the clinical observation, Cohen et al. mentioned certain soft (but not pathognomonic) pointers towards the diagnosis of COVID-19: i) shortness of breath between the $4^{\text {th }}$ and $8^{\text {th }}$ day of symptom onset, ii) breathlessness without productive cough, iii) sudden drop in oxygen saturation with minimal exertion [3]. Atypical symptoms like diarrhea or sudden loss of olfactory sensation are also been reported with COVID-19 [4].

Hence amidst the COVID-19 pandemic, it is important for the physicians to keep their approach streamlined and use appropriate laboratory tests and imaging studies to evaluate for the common diseases that can present with COVID-19 like symptoms. Common pulmonary diseases that can be confused with COVID-19 include
COPD exacerbation, bronchial asthma, interstitial lung disease, bacterial pneumonia, and pulmonary embolism [5]. Similarly, acute worsening of $\mathrm{CHF}$, acute coronary syndrome, pulmonary edema, hypertensive emergency, myocarditis, and cardiac arrhythmia are amongst the common cardiology COVID-19 mimickers. Notably, C reactive protein, D-dimer levels, cardiac enzymes, lactic acid dehydrogenase etc. can be elevated in most of these conditions, and as such there is no hallmark sign for COVID-19 pneumonia in lung imaging [6]. While the clinicians should be expeditious in diagnosing COVID-19, they should also be vigilant and always keep the other pulmonary, cardiac, or systemic differentials in mind because if missed, they could even lead to significantly higher consequences.

\section{References}

1. Sahu KK, Mishra AK, Lal A. COVID-2019: update on epidemiology, disease spread and management. Monaldi Arch Chest Dis 2020;90:1292. doi: 10.4081/monaldi.2020.1292.

2. Sahu KK, Lal A, Mishra AK. Latest updates on COVID-2019: A changing paradigm shift. J Med Virol 2020. doi: 10.1002 /jmv.25760.

3. Cohen PA, Hall L, John JN, Rapoport AB. The early natural history of SARS-CoV-2 infection: Clinical observations from an urban, ambulatory COVID-19 clinic. Mayo Clin Proc 2020. doi: 10.1016/j.mayocp.2020.04.010.

4. Eliezer M, Hautefort C, Hamel AL, et al. Sudden and complete olfactory loss function as a possible symptom of COVID-19. JAMA Otolaryngol Head Neck Surg 2020. doi: 10.1001/ jamaoto.2020.0832.

5. Cunha BA, Cunha CB. Legionnaire's disease and its mimics: a clinical perspective. Infect Dis Clin Nor Am 2017;31:95-109.

6. Sahu KK, Lal A, Mishra AK. An update on CT chest findings in coronavirus disease-19 (COVID-19). Heart Lung 2020. doi: 10.1016/j.hrtlng.2020.03.007. 